\title{
Nickel Oxide Nanoparticles Synthesized Under Microwave Irradiation
}

\author{
Armando Rodríguez, Boris Kharisov, Alejandro Vázquez \\ Universidad Autónoma de Nuevo León, UANL. Facultad de Ciencias Químicas \\ Av. Pedro de Alba S/N Cd. Universitaria 66450, San Nicolás de los Garza Nuevo León, México \\ mando1410@hotmail.com; bkhariss@mail.ru; alejandro.lqi@gmail.com
}

\section{Extended Abstract}

Nickel oxide is an important transition metal oxide which recently has received wide attention for its potential applications such as capacitors, electrochromics, lithium ion batteries, chemical sensors, and light-emitting diodes. Nickel oxide has a high stability and is a natural p type direct bandgap semiconductor, with a wide band gap $(3.4-4.0 \mathrm{eV})$, and a small electron affinity; also nickel is an abundant, low toxic transition metal. However, in order to enhance some of the properties of these devices, it is necessary to take this material to the nanometric scale. Several methods have been reported for the synthesis of nickel oxide nanoparticles such as sol-gel, spray pyrolysis, laser ablation, sonochemical, solvothermal and microwave-assisted methods. The synthesis of materials under microwave irradiation is a very promising research area because microwave chemistry is a fast synthetic technique with low energy consumption and high product yield. In this regard, the primary objective of this research was to develop a fast synthetic route of nickel oxide nanoparticles under microwave irradiation and investigate the effect of synthetic conditions on the material properties. The morphological, and chrystallographic characterization was the scope of this first stage of the research; investigation of potential applications such as light-emitting diode or lithium ion batteries is considered for a future work.

The synthesis of nickel oxide nanoparticles was developed in two stages: the first one was the synthesis of nickel hydroxide under microwave irradiation; and the second one was the heat treatment of nickel hydroxide in order to produce nickel oxide nanoparticles. Nickel hydroxide nanoparticles were prepared as follow, an ammonium hydroxide solution was employed to adjust the $\mathrm{pH}$ of a nickel chloride solution $(\mathrm{pH}=9$ and $\mathrm{pH}=10)$ which was heated under microwave irradiation $(t=60 \mathrm{~s}$ and $\mathrm{t}=90 \mathrm{~s})$. The effect of adding sodium citrate as stabilizer was also studied. The obtained precipitates were centrifuged and washed with deionized water and ethanol several times and were dried at $200{ }^{\circ} \mathrm{C}$ for $1 \mathrm{~h}$. The particles were analyzed by Dynamic Light Scattering to estimate the particle size, Scanning Electron Microscopy and X-ray Diffraction in order to characterize the morphology and chrystallographic phases of the product respectively. In order to obtain the nickel oxide nanoparticles, the nickel hydroxide powders were annealed at $400{ }^{\circ} \mathrm{C}$ for $2 \mathrm{~h}$ and analyzed by Scanning Electron Microscopy and X-ray Diffraction.

According to the XRD of the nickel hydroxide, the $\alpha$-phase hydroxide was obtained in the synthesis with the sodium citrate and the $\beta$-phase hydroxide was obtained in the synthesis without the sodium citrate. The DLS showed some particle sizes from 10 to $1000 \mathrm{~nm}$ depending of both the $\mathrm{pH}$ and the microwave irradiation time. After the annealing both of the polymorphs were successfully converted to nickel oxide in cubic form, the diffractograms were analyzed with the Scherrer equation obtaining a diameter of around $10 \mathrm{~nm}$ in all cases. 